\title{
Hohe Anforderungen an die Diagnostik - gute Therapieerfolge
}

\begin{abstract}
Das verbesserte Verständnis der dem Lungenkarzinom zugrunde liegenden genetischen Veränderungen und die Entwicklung zielgerichteter Therapien können die Effektivität der Behandlung erhöhen und den Verlauf der Erkrankung verbessern.
\end{abstract}

Ein Durchbruch bei der Behandlung von Patienten mit nicht-kleinzelligem Lungenkarzinom (NSCLC) könnte durch den personalisierten Einsatz zielgerichteter Therapeutika erreicht werden, erwartet Prof. Dr. Jürgen Wolf, Köln. Derzeit sind bei etwa
$50 \%$ der Adenokarzinome des NSCLC genetische Veränderungen bekannt, die Tumorwachstum und -vermehrung verstärken. Diese Veränderungen betreffen beispielsweise das EGFR (Epidermal Growth Factor Receptor)-Protoonkogen und das ALK (Anaplastic Lymphoma Kinase)-Gen und führen zu einer erhöhten Tyrosinkinaseaktivität. Mit personalisierten Therapieansätzen können laut Wolf substanzielle Fortschritte erreicht werden, die Umsetzung erfordere jedoch eine enge $\mathrm{Zu}$ sammenarbeit von Grundlagenforschern, Pathologen, Klinikern und Kostenträgern.

\section{ALK-Genfusion: neue Zielstruktur}

Durch eine Translokation auf Chromosom 2 fusioniert das ALK- mit dem EML4 (Echinoderm Microtubli Associated Protein Like 4)-Gen. Das EML4-ALK-Fusionsonkogen wurde bei 3-5\% der NSCLC-Patienten - zumeist jüngere Patienten und Nichtraucher nachgewiesen (Sasaki T et al., Eur J Cancer 2010, 46:1773-1780). Die konstitutiv aktivierte Rezeptortyrosinkinase führt zur erhöhten Tumorzellproliferation. Der in den USA bereits zugelassene ALK-Inhibitor Crizotinib führte bei Patienten mit ALK-positivem NSCLC zu Gesamtansprechraten von 54$61 \%$ und einem medianen progressionsfreien Überleben von 10 Monaten (Gandhi L, Jänne PA, Clin Cancer Res 2012, 18:37373742). $90 \%$ der Patienten erreichten eine Reduktion/Stabilisation des Tumors.

(aam)

Fachmedienveranstaltung von Pfizer Deutschland $\mathrm{GmbH}$

\section{Misteltherapie erzielt deutlichen Benefit}

Derzeit sind die therapeutischen Möglichkeiten bei Patienten mit späten Stadien des Pankreaskarzinoms deutlich begrenzt. Vielversprechende Ergebnisse ergab eine aktuelle Studie, in der mit der Gabe von Mistelextrakt sowohl die Überlebenszeit als auch die Lebensqualität der Patienten verbessert werden konnte.

Die palliative Therapie bei Patienten mit inoperablem Pankreaskarzinom ist weiterhin

verlängerung von wenigen Monaten erreichen.

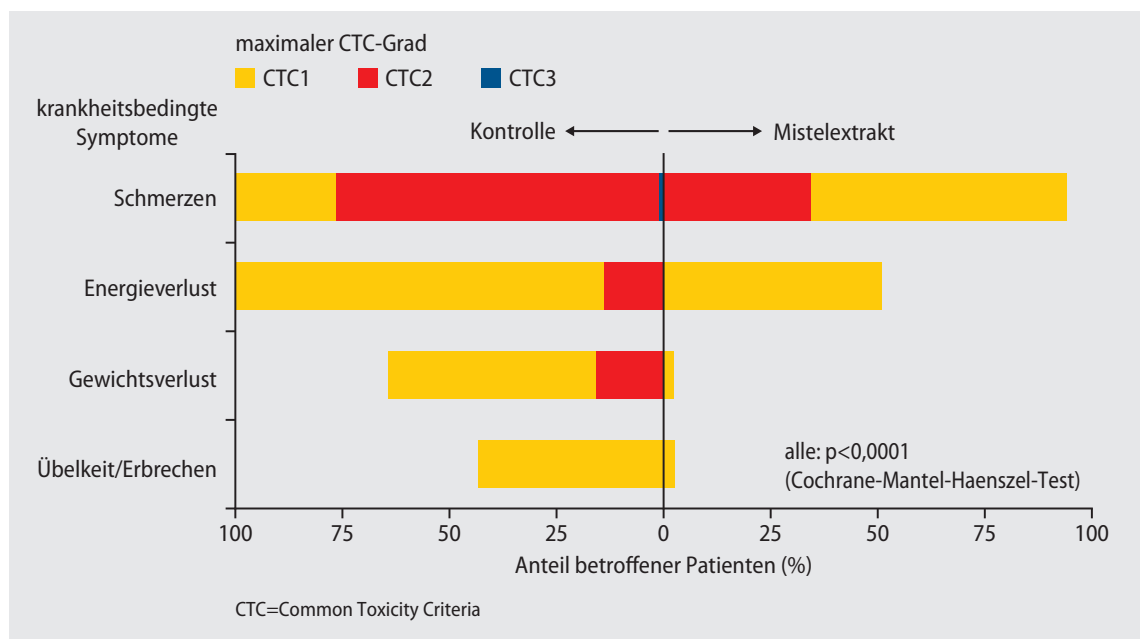

Reduktion krankheitsbedingter Symptome unter Misteltherapie bei Patienten mit Pankreaskarzinom (nach Galun D et al., Ann Oncol 2012, 23 [Suppl 9]:712)

unbefriedigend, so PD Dr. Harald Matthes, Berlin. Mit dem bisherigen Therapiestandard Gemcitabin lassen sich lediglich Ansprechraten von 15-30\% und eine Lebens-

\section{Längere Überlebenszeit}

Dass Patienten auch von einer alleinigen Misteltherapie profitieren, zeigten erste Ergebnisse einer prospektiven Phase-III-Stu- die in Serbien, die Dr. Marcus Reif, Berlin, vorstellte. Die Patienten - mit lokal fortgeschrittenem oder metastasiertem Pankreaskarzinom - erhielten über zwölf Monate randomisiert eine Best Supportive Care (BSC)-Behandlung (symptomatische Therapie gegen Schmerzen und gastroenterologische Beschwerden) mit oder ohne zusätzlichen Mistelextrakt (Iscador ${ }^{\circledast} \mathrm{Qu}$ spezial s.c., dreimal wöchentlich). Die Kontrollgruppe bekam keine weitere antineoplastische Therapie. Eine Zwischenanalyse mit Daten von 220 Patienten konnte nun eine alleinige Wirkung des Mistelpräparats zeigen. So überlebten die Patienten mit der Studienmedikation im Durchschnitt 2,1 Monate länger (4,8 versus 2,7 Monate) als im Kontrollarm. Selbst bei besonders ungünstiger Prognose (Metastasen, hohes Alter, schlechtes Allgemeinbefinden) zeigte sich noch ein Überlebensvorteil (2,0 versus 3,4 Monate). Den größten Benefit (7,2 versus 3,2 Monate) hatten Patienten ohne Metastasen. Dass 17 von 110 Verumpatienten die zwölfmonatige Studiendauer überlebt haben, wertete Reif ebenfalls als „, harten Parameter".

\section{Weniger tumorbedingte Symptome} Patienten, die das Mistelprodukt erhielten, berichteten zudem seltener von krankheitsassoziierten Symptomen, wie z.B. Fatigue, Übelkeit, Schmerzen, Appetitverlust, Schlaflosigkeit und Gewichtsverlust. (miu)

Experten-Round-Table der Weleda AG 